Internat. J. Math. \& Math. Sci.

Vol. 23, No. 11 (2000) 753-758

S0161171200003562

(C) Hindawi Publishing Corp.

\title{
ON CHARACTERIZATIONS OF A CENTER GALOIS EXTENSION
}

\author{
GEORGE SZETO and LIANYONG XUE
}

(Received 16 June 1999)

\begin{abstract}
Let $B$ be a ring with $1, C$ the center of $B, G$ a finite automorphism group of $B$, and $B^{G}$ the set of elements in $B$ fixed under each element in $G$. Then, it is shown that $B$ is a center Galois extension of $B^{G}$ (that is, $C$ is a Galois algebra over $C^{G}$ with Galois group $\left.G\right|_{C} \cong G$ ) if and only if the ideal of $B$ generated by $\{c-g(c) \mid c \in C\}$ is $B$ for each $g \neq 1$ in $G$. This generalizes the well known characterization of a commutative Galois extension $C$ that $C$ is a Galois extension of $C^{G}$ with Galois group $G$ if and only if the ideal generated by $\{c-g(c) \mid c \in C\}$ is $C$ for each $g \neq 1$ in $G$. Some more characterizations of a center Galois extension $B$ are also given.
\end{abstract}

Keywords and phrases. Galois extensions, center Galois extensions, central extensions, Galois central extensions, Azumaya algebras, separable extensions, $H$-separable extensions.

2000 Mathematics Subject Classification. Primary 16S30, 16W20.

1. Introduction. Let $C$ be a commutative ring with $1, G$ a finite automorphism group of $C$ and $C^{G}$ the set of elements in $C$ fixed under each element in $G$. It is well known that a commutative Galois extension $C$ is characterized in terms of the ideals generated by $\{c-g(c) \mid c \in C\}$ for $g \neq 1$ in $G$, that is $C$ is a Galois extension with Galois group $G$ if and only if the ideal generated by $\{c-g(c) \mid c \in C\}$ is $C$ for each $g \neq 1$ in $G$ (see [3, Proposition 1.2, page 80]). A natural generalization of a commutative Galois extension is the notion of a center Galois extension, that is, a noncommutative ring $B$ with a finite automorphism group $G$ and center $C$ is called a center Galois extension of $B^{G}$ with Galois group $G$ if $C$ is a Galois extension of $C^{G}$ with Galois group $\left.G\right|_{C} \cong G$. Ikehata (see $[4,5])$ characterized a center Galois extension with a cyclic Galois group $G$ of prime order in terms of a skew polynomial ring. Then, the present authors generalized the Ikehata characterization to center Galois extensions with Galois group $G$ of any cyclic order [7] and to center Galois extensions with any finite Galois group $G$ [8]. The purpose of the present paper is to generalize the above characterization of a commutative Galois extension to a center Galois extension. We shall show that $B$ is a center Galois extension of $B^{G}$ if and only if the ideal of $B$ generated by $\{c-g(c) \mid c \in C\}$ is $B$ for each $g \neq 1$ in $G$. A center Galois extension $B$ is also equivalent to each of the following statements:

(i) $B$ is a Galois central extension of $B^{G}$, that is, $B=B^{G} C$ which is $G$-Galois extension of $B^{G}$.

(ii) $B$ is a Galois extension of $B^{G}$ with a Galois system $\left\{b_{i} \in B, c_{i} \in C, i=1,2, \ldots, m\right\}$ for some integer $m$.

(iii) the ideal of the subring $B^{G} C$ generated by $\{c-g(c) \mid c \in C\}$ is $B^{G} C$ for each $g \neq 1$ in $G$. 
2. Definitions and notations. Throughout this paper, $B$ will represent a ring with $1, G=\left\{g_{1}=1, g_{2}, \ldots, g_{n}\right\}$ an automorphism group of $B$ of order $n$ for some integer $n, C$ the center of $B, B^{G}$ the set of elements in $B$ fixed under each element in $G$, and $B * G$ a skew group ring in which the multiplication is given by $g b=g(b) g$ for $b \in$ $B$ and $g \in G$.

$B$ is called a $G$-Galois extension of $B^{G}$ if there exist elements $\left\{a_{i}, b_{i} \in B, i=1,2, \ldots, m\right\}$ for some integer $m$ such that $\sum_{i=1}^{m} a_{i} g\left(b_{i}\right)=\delta_{1, g}$. Such a set $\left\{a_{i}, b_{i}\right\}$ is called a $G$-Galois system for $B . B$ is called a center Galois extension of $B^{G}$ if $C$ is a Galois algebra over $C^{G}$ with Galois group $\left.G\right|_{C} \cong G$. $B$ is called a central extension of $B^{G}$ if $B=B^{G} C$, and $B$ is called a Galois central extension of $B^{G}$ if $B=B^{G} C$ is a Galois extension of $B^{G}$ with Galois group $G$.

Let $A$ be a subring of a ring $B$ with the same identity 1 . We denote $V_{B}(A)$ the commutator subring of $A$ in $B$. We call $B$ a separable extension of $A$ if there exist $\left\{a_{i}, b_{i} \in B, i=1,2, \ldots, m\right.$ for some integer $\left.m\right\}$ such that $\sum a_{i} b_{i}=1$, and $\sum b a_{i} \otimes b_{i}=$ $\sum a_{i} \otimes b_{i} b$ for all $b \in B$ where $\otimes$ is over $A$. $B$ is called $H$-separable extension of $A$ if $B \otimes_{A} B$ is isomorphic to a direct summand of a finite direct sum of $B$ as a $B$-bimodule. $B$ is called centrally projective over $A$ if $B$ is a direct summand of a finite direct sum of $A$ as a $A$-bimodule.

3. The characterizations. In this section, we denote $J_{j}^{(C)}=\left\{c-g_{j}(c) \mid c \in C\right\}$. We shall show that $B$ is a center Galois extension of $B^{G}$ if and only if $B=B J_{j}^{(C)}$, the ideal of $B$ generated by $J_{j}^{(C)}$, for each $g_{j} \neq 1$ in $G$. Some more characterizations of a center Galois extension $B$ are also given. We begin with a lemma.

LEMMA 3.1. If $B=B J_{j}^{(C)}$ for each $g_{j} \neq 1$ in $G$ (that is, $j \neq 1$ ), then

(1) $B$ is a Galois extension of $B^{G}$ with Galois group $G$ and a Galois system $\left\{b_{i} \in B ; c_{i} \in\right.$ $C, i=1,2, \ldots, m\}$ for some integer $m$.

(2) $B$ is a centrally projective over $B^{G}$.

(3) $B * G$ is $H$-separable over $B$.

(4) $V_{B * G}(B)=C$.

Proof. (1) Since $B=B J_{j}^{(C)}$ for each $j \neq 1$, there exist $\left\{b_{i}^{(j)} \in B, c_{i}^{(j)} \in C, i=\right.$ $\left.1,2, \ldots, m_{j}\right\}$ for some integer $m_{j}, j=2,3, \ldots, n$ such that $\sum_{i=1}^{m_{j}} b_{i}^{(j)}\left(c_{i}^{(j)}-g_{j}\left(c_{i}^{(j)}\right)\right)=1$. Therefore, $\sum_{i=1}^{m_{j}} b_{i}^{(j)} c_{i}^{(j)}=1+\sum_{i=1}^{m_{j}} b_{i}^{(j)} g_{j}\left(c_{i}^{(j)}\right)$. Let $b_{m_{j}+1}^{(j)}=-\sum_{i=1}^{m_{j}} b_{i}^{(j)} g_{j}\left(c_{i}^{(j)}\right)$ and $c_{m_{j}+1}^{(j)}=1$. Then $\sum_{i=1}^{m_{j}+1} b_{i}^{(j)} c_{i}^{(j)}=1$ and $\sum_{i=1}^{m_{j}+1} b_{i}^{(j)} g_{j}\left(c_{i}^{(j)}\right)=0$. Let $b_{i_{2}, i_{3}, \ldots, i_{n}}=b_{i_{2}}^{(2)} b_{i_{3}}^{(3)}$ $\cdots b_{i n}^{(n)}$ and $c_{i_{2}, i_{3}, \ldots, i_{n}}=c_{i_{2}}^{(2)} c_{i_{3}}^{(3)} \ldots c_{i_{n}}^{(n)}$ for $i_{j}=1,2, \ldots, m_{j}+1$ and $j=2,3, \ldots, n$. Then

$$
\begin{aligned}
\sum_{i_{2}=1}^{m_{2}+1} \sum_{i_{3}=1}^{m_{3}+1} \cdots \sum_{i_{n}=1}^{m_{n}+1} b_{i_{2}, i_{3}, \ldots, i_{n}} c_{i_{2}, i_{3}, \ldots, i_{n}} & =\sum_{i_{2}=1}^{m_{2}+1} \sum_{i_{3}=1}^{m_{3}+1} \cdots \sum_{i_{n}=1}^{m_{n}+1} b_{i_{2}}^{(2)} b_{i_{3}}^{(3)} \cdots b_{i_{n}}^{(n)} c_{i_{2}}^{(2)} c_{i_{3}}^{(3)} \cdots c_{i_{n}}^{(n)} \\
& =\sum_{i_{2}=1}^{m_{2}+1} \sum_{i_{3}=1}^{m_{3}+1} \cdots \sum_{i_{n}=1}^{m_{n}+1} b_{i_{2}}^{(2)} c_{i_{2}}^{(2)} b_{i_{3}}^{(3)} c_{i_{3}}^{(3)} \cdots b_{i_{n}}^{(n)} c_{i_{n}}^{(n)} \\
& =\sum_{i_{2}=1}^{m_{2}+1} b_{i_{2}}^{(2)} c_{i_{2}}^{(2)} \sum_{i_{3}=1}^{m_{3}+1} b_{i_{3}}^{(3)} c_{i_{3}}^{(3)} \cdots \sum_{i_{n}=1}^{m_{n}+1} b_{i_{n}}^{(n)} c_{i_{n}}^{(n)}=1
\end{aligned}
$$


and for each $j \neq 1$

$$
\begin{aligned}
\sum_{i_{2}=1}^{m_{2}+1} \sum_{i_{3}=1}^{m_{3}+1} \cdots \sum_{i_{n}=1}^{m_{n}+1} b_{i 2, i_{3}, \ldots, i_{n}} g_{j}\left(c_{i_{2}, i_{3}, \ldots, i_{n}}\right) \\
\quad=\sum_{i_{2}=1}^{m_{2}+1} \sum_{i_{3}=1}^{m_{3}+1} \cdots \sum_{i_{n}=1}^{m_{n}+1} b_{i_{2}}^{(2)} b_{i_{3}}^{(3)} \cdots b_{i_{n}}^{(n)} g_{j}\left(c_{i_{2}}^{(2)} c_{i_{3}}^{(3)} \cdots c_{i_{n}}^{(n)}\right) \\
=\sum_{i_{2}=1}^{m_{2}+1} \sum_{i_{3}=1}^{m_{3}+1} \cdots \sum_{i_{n}=1}^{m_{n}+1} b_{i_{2}}^{(2)} b_{i_{3}}^{(3)} \cdots b_{i_{n}}^{(n)} g_{j}\left(c_{i_{2}}^{(2)}\right) g_{j}\left(c_{i_{3}}^{(3)}\right) \cdots g_{j}\left(c_{i_{n}}^{(n)}\right) \\
=\sum_{i_{2}=1}^{m_{2}+1} \sum_{i_{3}=1}^{m_{3}+1} \cdots \sum_{i_{n}=1}^{m_{n}+1} b_{i_{2}}^{(2)} g_{j}\left(c_{i_{2}}^{(2)}\right) b_{i_{3}}^{(3)} g_{j}\left(c_{i_{3}}^{(3)}\right) \cdots b_{i_{n}}^{(n)} g_{j}\left(c_{i_{n}}^{(n)}\right) \\
=\sum_{i_{2}=1}^{m_{2}+1} b_{i_{2}}^{(2)} g_{j}\left(c_{i_{2}}^{(2)}\right) \sum_{i_{3}=1}^{m_{3}+1} b_{i_{3}}^{(3)} g_{j}\left(c_{i_{3}}^{(3)}\right) \cdots \sum_{i_{n}=1}^{m_{n}+1} b_{i_{n}}^{(n)} g_{j}\left(c_{i_{n}}^{(n)}\right)=0 .
\end{aligned}
$$

Thus, $\left\{b_{i_{2}, i_{3}, \ldots, i_{n}} \in B ; c_{i_{2}, i_{3}, \ldots, i_{n}} \in C, i_{j}=1,2, \ldots, m_{j}+1\right.$ and $\left.j=2,3, \ldots, n\right\}$ is a Galois system for $B$. This complete the proof of (1).

(2) By (1), $B$ is a Galois extension of $B^{G}$ with a Galois system $\left\{b_{i} \in B, c_{i} \in C, i=\right.$ $1,2, \ldots, m\}$ for some integer $m$. Let $f_{i}: B \rightarrow B^{G}$ given by $f_{i}(b)=\sum_{j=1}^{n} g_{j}\left(c_{i} b\right)$ for all $b \in$ $B, i=1,2, \ldots, m$. Then it is easy to check that $f_{i}$ is a homomorphism as $B^{G}$-bimodule and $b=\sum_{i=1}^{m} b_{i} c_{i} b=\sum_{j=1}^{n} \sum_{i=1}^{m} b_{i} g_{j}\left(c_{i}\right) g_{j}(b)=\sum_{i=1}^{m} b_{i} \sum_{j=1}^{n} g_{j}\left(c_{i} b\right)=\sum_{i=1}^{m} b_{i} f_{i}(b)$ for all $b \in B$. Hence $\left\{b_{i} ; f_{i}, i=1,2, \ldots, m\right\}$ is a dual bases for $B$ as $B^{G}$-bimodule, and so $B$ is finitely generated and projective as $B^{G}$-bimodule. Therefore, $B$ is a direct summand of a finite direct sum of $B^{G}$ as a $B^{G}$-bimodule. Thus $B$ is centrally projective over $B^{G}$.

(3) By (1), $B$ is a Galois extension of $B^{G}$ with Galois group $G$. Hence $B * G \cong \operatorname{Hom}_{B} G(B$, $B$ ) $\left[2\right.$, Theorem 1]. By (2), $B$ is centrally projective over $B^{G}$. Thus, $B * G\left(\cong \operatorname{Hom}_{B}(B, B)\right)$ is $H$-separable over $B$ [6, Proposition 11].

(4) We first claim that $V_{B * G}(C)=B$. Clearly, $B \subset V_{B * G}(C)$. Let $\sum_{j=1}^{n} b_{j} g_{j}$ in $V_{B * G}(C)$ for some $b_{j} \in B$. Then $c\left(\sum_{j=1}^{n} b_{j} g_{j}\right)=\left(\sum_{j=1}^{n} b_{j} g_{j}\right) c$ for each $c \in C$, so $c b_{j}=b_{j} g_{j}(c)$, that is, $b_{j}\left(c-g_{j}(c)\right)=0$ for each $g_{j} \in G$ and $c \in C$. Since $B=B J_{j}^{(C)}$ for each $g_{j} \neq 1$, there exist $b_{i}^{(j)} \in B$ and $c_{i}^{(j)} \in C, i=1,2, \ldots, m$ such that $\sum_{i=1}^{m} b_{i}^{(j)}\left(c_{i}^{(j)}-g_{j}\left(c_{i}^{(j)}\right)\right)=$ 1. Hence $b_{j}=\sum_{i=1}^{m} b_{i}^{(j)}\left(c_{i}^{(j)}-g_{j}\left(c_{i}^{(j)}\right)\right) b_{j}=\sum_{i=1}^{m} b_{i}^{(j)} b_{j}\left(c_{i}^{(j)}-g_{j}\left(c_{i}^{(j)}\right)\right)=0$ for each $g_{j} \neq 1$. This implies that $\sum_{j=1}^{n} b_{j} g_{j}=b_{1} \in B$. Hence $V_{B * G}(C) \subseteq B$, and so $V_{B * G}(C)=B$. Therefore, $V_{B * G}(B) \subset V_{B * G}(C)=B$. Thus $V_{B * G}(B)=V_{B}(B)=C$.

We now show some characterizations of a center Galois extension $B$.

THEOREM 3.2. The following statements are equivalent.

(1) $B$ is a center Galois extension of $B^{G}$.

(2) $B=B J_{j}^{(C)}$ for each $g_{j} \neq 1$ in $G$.

(3) $B$ is a Galois extension of $B^{G}$ with a Galois system $\left\{b_{i} \in B, c_{i} \in C, i=1,2, \ldots, m\right\}$ for some integer $m$.

(4) $B$ is a Galois central extension of $B^{G}$.

(5) $B^{G} C=B^{G} C J_{j}^{(C)}$ for each $g_{j} \neq 1$ in $G$. 
Proof. $(1) \Longrightarrow(2)$. By hypothesis, $C$ is a Galois extension of $C^{G}$ with Galois group $\left.G\right|_{C} \cong G$. Hence $C=C J_{j}^{(C)}$ for each $g_{j} \neq 1$ in $G$ [3, Proposition 1.2, page 80]. Thus, $B=B J_{j}^{(C)}$ for each $g_{j} \neq 1$ in $G$.

(2) $\Longrightarrow(1)$. Since $B=B J_{j}^{C}$ for each $g_{j} \neq 1$ in $G, B * G$ is $H$-separable over $B$ by Lemma 3.1(3) and $V_{B * G}(B)=C$ by Lemma 3.1(4). Thus $C$ is a Galois extension of $C^{G}$ with Galois group $\left.G\right|_{C} \cong G$ by [1, Proposition 4].

$(1) \Longrightarrow(3)$. This is Lemma 3.1(1).

$(3) \Longrightarrow(1)$. Since $B$ is Galois extension of $B^{G}$ with a Galois system $\left\{b_{i} \in B, c_{i} \in C, i=\right.$ $1,2, \ldots, m\}$ for some integer $m$, we have $\sum_{i=1}^{m} b_{i} g_{j}\left(c_{i}\right)=\delta_{1, g}$. Hence $\sum_{i=1}^{m} b_{i}\left(c_{i}-g_{j}\left(c_{i}\right)\right)$ $=1$ for each $g_{j} \neq 1$ in $G$. So for every $b \in B, b=\sum_{i=1}^{m} b b_{i}\left(c_{i}-g_{j}\left(c_{i}\right)\right) \in B J_{j}^{(C)}$. Therefore, $B=B J_{i}^{(C)}$ for each $g_{i} \neq 1$ in $G$. Thus, $B$ is a center Galois extension of $B^{G}$ by $(2) \Longrightarrow(1)$.

$(1) \Longrightarrow(4)$. Since $C$ is a Galois algebra with Galois group $\left.G\right|_{C} \cong G, B$ and $B^{G} C$ are Galois extensions of $B^{G}$ with Galois group $\left.G\right|_{B^{G} C} \cong G$. Noting that $B^{G} C \subset B$, we have $B=B^{G} C$, that is, $B$ is a central extension of $B^{G}$. But $B$ is a Galois extension of $B^{G}$, so $B$ is a Galois central extension of $B^{G}$.

(4) $\Longrightarrow(1)$. By hypothesis, $B=B^{G} C$ is a Galois extension of $B^{G}$. Hence there exists a Galois system $\left\{a_{i} ; b_{i} \in B, i=1,2, \ldots, m\right\}$ for some integer $m$ such that $\sum_{i=1}^{m} a_{i} g_{j}\left(b_{i}\right)=$ $\delta_{1, j}$. But $B=B^{G} C$, so $a_{i}=\sum_{k=1}^{n_{a_{i}}} b_{k}^{\left(a_{i}\right)} c_{k}^{\left(a_{i}\right)}$ and $b_{i}=\sum_{l=1}^{n_{b_{i}}} b_{l}^{\left(b_{i}\right)} c_{l}^{\left(b_{i}\right)}$ for some $a_{k}^{\left(a_{i}\right)}, b_{l}^{\left(b_{i}\right)}$ in $B^{G}$ and $c_{k}^{\left(a_{i}\right)}, c_{l}^{\left(b_{i}\right)}$ in $C, k=1,2 \ldots, n_{a_{i}}, l=1,2, \ldots, n_{b_{i}}, i=1,2, \ldots, m$. Therefore,

$$
\begin{aligned}
\delta_{1, j} & =\sum_{i=1}^{m} a_{i} \mathcal{g}_{j}\left(b_{i}\right)=\sum_{i=1}^{m} \sum_{k=1}^{n_{a_{i}}} b_{k}^{\left(a_{i}\right)} c_{k}^{\left(a_{i}\right)} g_{j}\left(\sum_{l=1}^{n_{b_{i}}} b_{l}^{\left(b_{i}\right)} c_{l}^{\left(b_{i}\right)}\right) \\
& =\sum_{i=1}^{m} \sum_{k=1}^{n_{a_{i}}} b_{k}^{\left(a_{i}\right)} c_{k}^{\left(a_{i}\right)} \sum_{l=1}^{n_{b_{i}}} b_{l}^{b_{i}} g_{j}\left(c_{l}^{\left(b_{i}\right)}\right)=\sum_{i=1}^{m} \sum_{k=1}^{n_{a_{i}}} \sum_{l=1}^{n_{b_{i}}}\left(b_{k}^{\left(a_{i}\right)} c_{k}^{\left(a_{i}\right)} b_{l}^{\left(b_{i}\right)}\right) g_{j}\left(c_{l}^{\left(b_{i}\right)}\right) .
\end{aligned}
$$

This shows that $\left\{b_{k, l}^{\left(a_{i}, b_{i}\right)}=b_{k}^{\left(a_{i}\right)} c_{k}^{\left(a_{i}\right)} b_{l}^{\left(b_{i}\right)} \in B ; c_{k, l}^{\left(a_{i}, b_{i}\right)}=c_{l}^{\left(b_{i}\right)} \in C, k=1,2, \ldots, n_{a_{i}}, l=\right.$ $\left.1,2, \ldots, n_{b_{i}}, i=1,2, \ldots, m\right\}$ is a Galois system for $B$. Thus, $B$ is a center Galois extension of $B^{G}$ by $(3) \Longrightarrow(1)$.

$(1) \Longrightarrow(5)$. Since $B$ is a center Galois extension of $B^{G}, B=B J_{j}^{(C)}$ for each $g_{j} \neq 1$ in $G$ by $(1) \Longrightarrow(2)$ and $B=B^{G} C$ by (1) $\Longrightarrow(4)$. Thus, $B^{G} C=B^{G} C J_{j}^{(C)}$ for each $g_{j} \neq 1$ in $G$.

(5) $\Longrightarrow(1)$. Since $B^{G} C=B^{G} C J_{j}^{(C)}$ for each $g_{j} \neq 1$ in $G, B=B J_{j}^{(C)}$ for each $g_{j} \neq 1$ in $G$. Thus, $B$ is a center Galois extension of $B^{G}$ by (2) $\Longrightarrow(1)$.

The characterization of a commutative Galois extension $C$ in terms of the ideals generated by $\{c-g(c) \mid c \in C\}$ for $g \neq 1$ in $G$ is an immediate consequence of Theorem 3.2.

COROLLARY 3.3. A commutative ring $C$ is a Galois extension of $C^{G}$ if and only if $C=C J_{j}^{(C)}$, the ideal generated by $\left\{c-g_{j}(c) \mid c \in C\right\}$ is $C$ for each $g_{j} \neq 1$ in $G$.

Proof. Let $B=C$ in Theorem 3.2. Then, the corollary is an immediate consequence of Theorem 3.2(2).

By Theorem 3.2, we derive several characterizations of a Galois centeral extension $B$. 
COROLLARY 3.4. If $B$ is a central extension of $B^{G}$ (that is, $B=B^{G} C$ ), then the following statements are equivalent.

(1) $B$ is a Galois extension of $B^{G}$.

(2) $B$ is a center Galois extension of $B^{G}$.

(3) $B * G$ is $H$-separable over $B$.

(4) $B=C J_{j}^{(B)}$ for each $g_{j} \neq 1$ in $G$.

(5) $B=B J_{j}^{(B)}$ for each $g_{j} \neq 1$ in $G$.

Proof. $(1) \Longleftrightarrow(2)$. This is given by $(1) \Longleftrightarrow(4)$ in Theorem 3.2 .

$(2) \Longrightarrow(3)$. This is Lemma 3.1(3).

$(3) \Longrightarrow(1)$. Since $B * G$ is $H$-separable over $B, B$ is a Galois extension of $B^{G}$ [1, Proposition 2].

Since $B=B^{G} C$ by hypothesis, it is easy to see that $J_{j}^{(B)}=B^{G} J_{j}^{(C)}$ for each $g_{j}$ in $G$. Thus, $B=C J_{j}^{(B)}, B=B J_{j}^{(B)}$, and $B=B J_{j}^{(C)}$ are equivalent. This implies that $(2) \Longleftrightarrow(4) \Longleftrightarrow(5)$ by Theorem 3.2(2).

We call a ring $B$ the DeMeyer-Kanzaki Galois extension of $B^{G}$ if $B$ is an Azumaya $C$ algebra and $B$ is a center Galois extension of $B^{G}$ (for more about the DeMeyer-Kanzaki Galois extensions, see [2]). Clearly, the class of center Galois extensions is broader than the class of the DeMeyer-Kanzaki Galois extensions. We conclude the present paper with two examples. (1) The DeMeyer-Kanzaki Galois extension of $B^{G}$ and (2) a center Galois extension of $B^{G}$, but not the DeMeyer-Kanzaki Galois extension of $B^{G}$.

EXAMPLE 3.5. Let $\mathbb{C}$ be the field of complex numbers, that is, $\mathbb{C}=\mathbb{R}+\mathbb{R} \sqrt{-1}$ where $\mathbb{R}$ is the field of real numbers, $B=\mathbb{C}[i, j, k]$ the quaternion algebra over $\mathbb{C}$, and $G=$ $\left\{1, g \mid g\left(c_{1}+c_{i} i+c_{j} j+c_{k} k\right)=g\left(c_{1}\right)+g\left(c_{i}\right) i+g\left(c_{j}\right) j+g\left(c_{k}\right) k\right.$ for each $b=c_{1}+c_{i} i+$ $c_{j} j+c_{k} k \in \mathbb{C}[i, j, k]$ and $g(u+v \sqrt{-1})=u-v \sqrt{-1}$ for each $\left.c=u+v \sqrt{-1} \in \mathbb{C}\right\}$. Then

(1) The center of $B$ is $\mathbb{C}$.

(2) $B$ is an Azumaya $C$-algebra.

(3) $\mathbb{C}$ is a Galois extension of $\mathbb{C}^{G}$ with Galois group $\left.G\right|_{\mathbb{C}} \cong G$ and a Galois system $\left\{a_{1}=1 / \sqrt{2}, a_{2}=(1 / \sqrt{2}) \sqrt{-1} ; b_{1}=1 / \sqrt{2}, b_{2}=-(1 / \sqrt{2}) \sqrt{-1}\right\}$.

(4) $B$ is the DeMeyer-Kanzaki Galois extension of $B^{G}$ by (2) and (3).

(5) $B^{G}=\mathbb{R}[i, j, k]$.

(6) $B=B^{G} \mathbb{C}$, so $B$ is a centeral extension of $B^{G}$.

(7) $J_{g}^{(\mathbb{C})}=\mathbb{R} \sqrt{-1}$.

(8) $B=B J_{\mathcal{g}}^{(C)}$ since $1=-\sqrt{-1} \sqrt{-1} \in B J_{g}^{(C)}$.

(9) $J_{\mathfrak{g}}^{(B)}=\mathbb{R} \sqrt{-1}+\mathbb{R} \sqrt{-1} i+\mathbb{R} \sqrt{-1} j+\mathbb{R} \sqrt{-1} k$.

(10) $B=\mathbb{C} J_{g}^{(B)}$.

EXAMPLE 3.6. By replacing in Example 3.5 the field of complex numbers $\mathbb{C}$ with the ring $C=\mathbb{Z} \oplus \mathbb{Z}$ where $\mathbb{Z}$ is the ring of integers, $g(a, b)=(b, a)$ for all $(a, b) \in C$, and $G=\left\{1, g \mid g\left(c_{1}+c_{i} i+c_{j} j+c_{k} k\right)=g\left(c_{1}\right)+g\left(c_{i}\right) i+g\left(c_{j}\right) j+g\left(c_{k}\right) k\right.$ for each $b=$ $\left.c_{1}+c_{i} i+c_{j} j+c_{k} k \in B=C[i, j, k]\right\}$. Then

(1) The center of $B$ is $C$.

(2) $C$ is a Galois extension of $C^{G}$ with Galois group $\left.G\right|_{C} \cong G$ and a Galois system $\left\{a_{1}=(1,0), a_{2}=(0,1) ; b_{1}=(1,0), b_{2}=(0,1)\right\}$. 
(3) $B$ is not an Azumaya $C$-algebra (for $1 / 2 \notin C$ ), and so $B$ is not the DeMeyer-Kanzaki Galois extension of $B^{G}$.

(4) $C^{G}=\{(a, a) \mid a \in \mathbb{Z}\} \cong \mathbb{Z}$.

(5) $B^{G}=C^{G}[i, j, k]$.

(6) $B=B^{G} C$, so $B$ is a central extension of $B^{G}$.

(7) $J_{\mathcal{g}}^{(C)}=\{(a,-a) \mid a \in \mathbb{Z}\}=\mathbb{Z}(1,-1)$.

(8) $B=B J_{g}^{(C)}$ since $1=(1,1)=(1,-1)(1,-1) \in B J_{G}^{(C)}$.

(9) $J_{g}^{(B)}=\mathbb{Z}(1,-1)+\mathbb{Z}(1,-1) i+\mathbb{Z}(1,-1) j+\mathbb{Z}(1,-1) k$.

(10) $B=C J_{g}^{(B)}$.

\section{REFERENCES}

[1] R. Alfaro and G. Szeto, The centralizer on H-separable skew group rings, Rings, extensions, and cohomology (Evanston, IL, 1993) (New York), Dekker, 1994, pp. 1-7. MR 95g:16027. Zbl 812.16038.

[2] F. R. DeMeyer, Some notes on the general Galois theory of rings, Osaka J. Math. 2 (1965), 117-127. MR 32\#128. Zbl 143.05602.

[3] F. R. DeMeyer and E. Ingraham, Separable Algebras over Commutative Rings, SpringerVerlag, Berlin, 1971. MR 43\#6199. Zbl 215.36602.

[4] S. Ikehata, On H-separable polynomials of prime degree, Math. J. Okayama Univ. 33 (1991), 21-26. MR 93g:16043. Zbl 788.16022.

[5] S. Ikehata and G. Szeto, On H-skew polynomial rings and Galois extensions, Rings, extensions, and cohomology (Evanston, IL, 1993) (New York), Dekker, 1994, pp. 113-121. MR 95j:16033. Zbl 815.16009.

[6] K. Sugano, Note on separability of endomorphism rings, J. Fac. Sci. Hokkaido Univ. Ser. I 21 (1970/71), 196-208. MR 45\#3465. Zbl 236.16003.

[7] G. Szeto and L. Xue, On the Ikehata theorem for H-separable skew polynomial rings, Math. J. Okayama Univ. 40 (1998), 27-32, 2000.

[8] _ The general Ikehata theorem for H-separable crossed products, Internat. J. Math. Math. Sci., Vol 25, to appear, 1999.

Szeto: Department of MAthematics, Bradley University, Peoria, Illinois 61625, USA

E-mail address: szeto@brad7ey.brad7ey.edu

Xue: Department of MAthematics, Bradley University, Peoria, Illinois 61625, USA

E-mail address: 1xue@brad7 ey.brad7ey.edu 


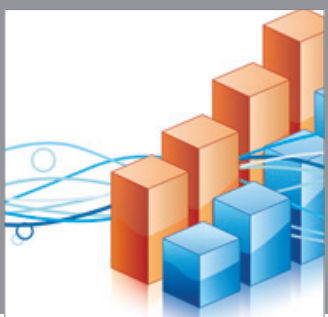

Advances in

Operations Research

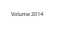

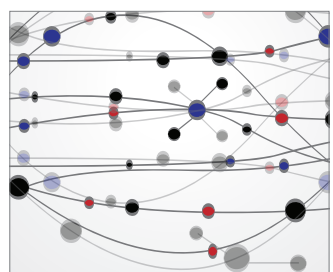

\section{The Scientific} World Journal
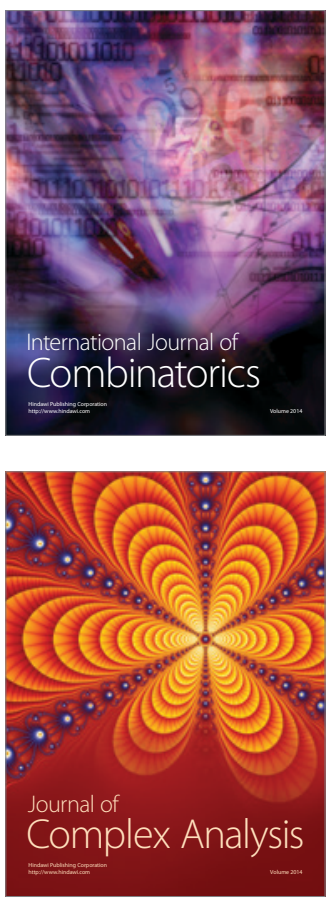

International Journal of

Mathematics and

Mathematical

Sciences
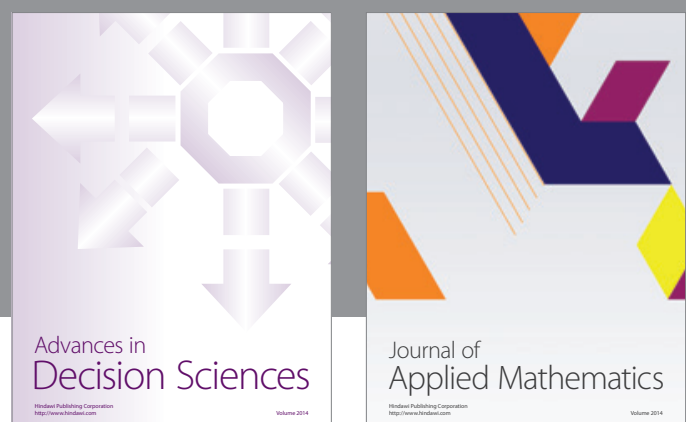

Journal of

Applied Mathematics
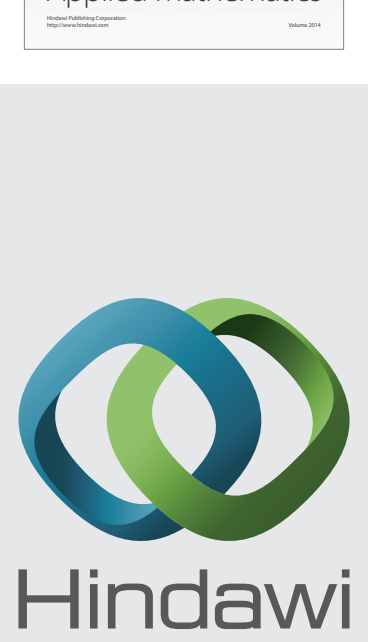

Submit your manuscripts at http://www.hindawi.com
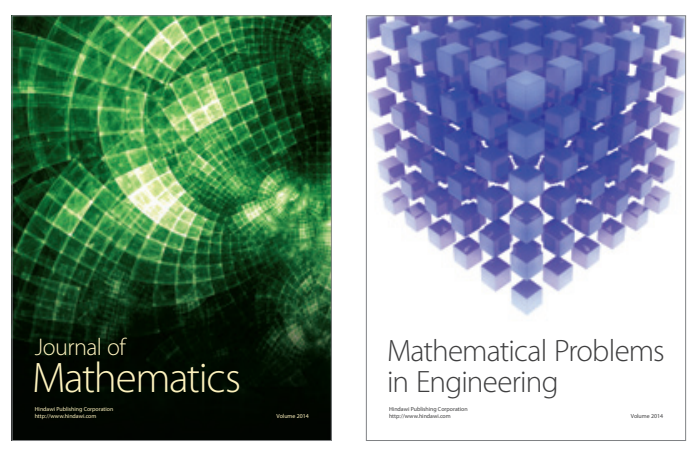

Mathematical Problems in Engineering
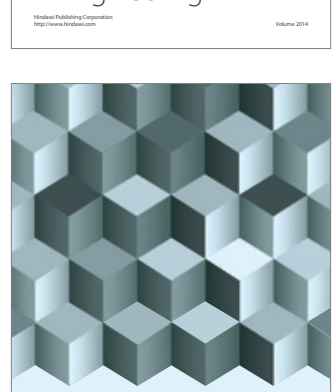

Journal of

Function Spaces
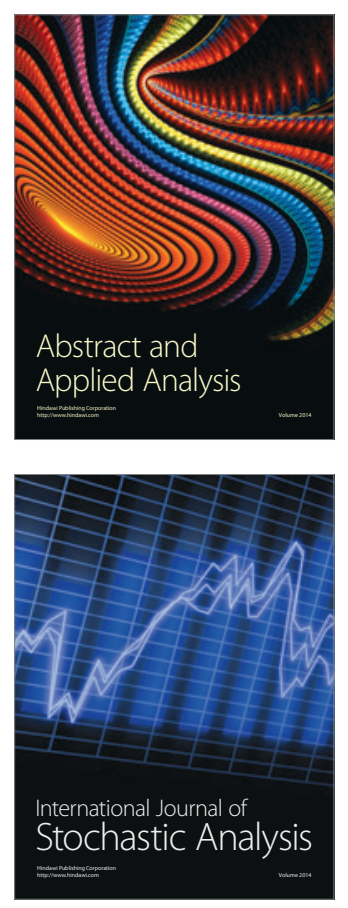

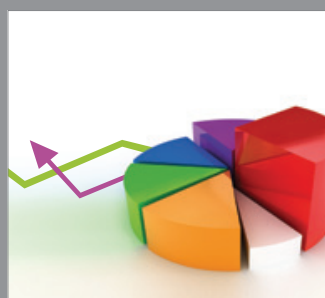

ournal of

Probability and Statistics

Promensencen
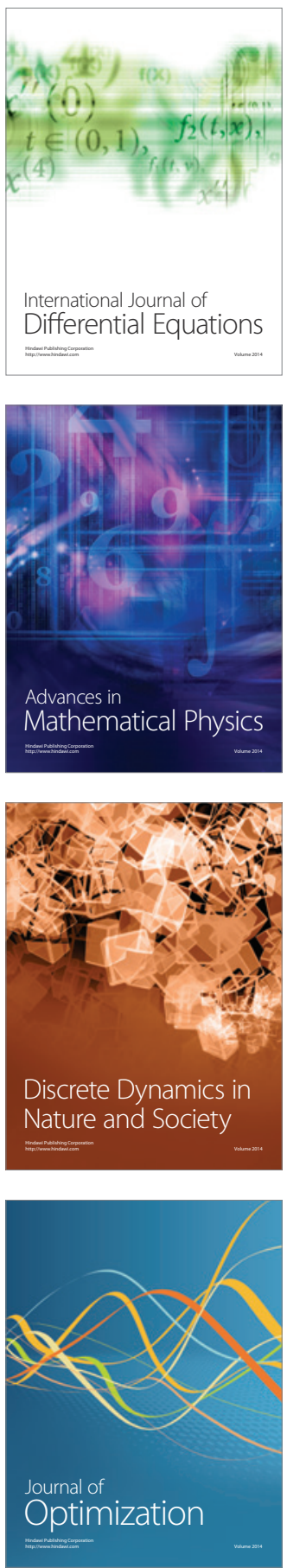